\title{
Predicting neutrino parameters from texture zeros
}

\author{
Alejandro Ibarra* ${ }^{* a}$ and Graham. G. Ross ${ }^{a, b}$ \\ a Theory Division, CERN, CH-1211 Geneva 23, Switzerland \\ ${ }^{b}$ Department of Physics, Theoretical Physics, University of Oxford, \\ 1 Keble Road, Oxford OX1 3NP, U.K. \\ E-mail: 'Álejandro. Ibarra@cern.ch, 'g.rossi@physics.ox.ac.uk
}

\begin{abstract}
The see-saw mechanism is perhaps the most attractive scenario to explain the small neutrino masses. However, at high energies it is defined by more parameters than it is possible to measure at low energies, resulting in a lack of predictability. In this talk we will review some frameworks where, using some well motivated hypotheses about the high energy physics, the see-saw mechanism does give predictions for some low energy observables. In particular, we will derive a leptonic version of the Gatto-Sartori-Tonin relation, and we will discuss the scenario with two right-handed neutrinos.
\end{abstract}

\section{Introduction}

The origin of the structure observed in the quark and lepton masses and mixing angles is one of the most intriguing features of the Standard Model of particle physics. Despite the large amount of data available, there is no compelling explanation for this structure. However, in the quark sector there are interesting ideas that could shed some light on the origin of flavour. Particularly attractive and robust is the postulate of texture zeros in the quark mass matrices. Assuming that the up and down quark mass matrices have a simultaneous texture zero in the $(1,1)$ position $^{1}$ and that the magnitude of the matrix elements are symmetric for the first two generations, yields a quantitatively accurate prediction for the Cabbibo angle (strictly speaking, for $V_{12}^{C K M}$ ). This is the so-called Gatto-Sartori-Tonin (GST) relation [i]. The measured masses and mixing angles are consistent with additional texture zeros $[\overline{2}]$, although this may require a departure from the symmetric form of the mass matrices [i]. These texture zeros could indicate the presence of an underlying family symmetry which require some matrix elements be anomalously small.

\footnotetext{
${ }^{*}$ Speaker.

${ }^{1} \mathrm{~A}$ texture zero does not imply a matrix element is absolutely zero, but only that it is small enough so that it does not significantly affect the masses and mixing angles.
} 
In this work we extend the analysis of texture zeros to the lepton sector, for the case in which the small neutrino masses are generated by the see-saw mechanism [4]- Motivated by the success of the Gatto-Sartori-Tonin relation in the quark sector, we will consider the predictions from texture zeros in the neutrino Yukawa coupling.

We will also study the model with two right-handed neutrinos proposed by Frampton, Glashow and Yanagida [5.5. This model provides a framework that can accommodate all the neutrino observations, but with less parameters than the complete model with three right handed neutrinos.

\section{From low to high energies}

We consider the case of three generations of left-handed $S U(2)$ doublet neutrinos, $\nu_{L, i}$, and three generations of right-handed Standard Model singlet neutrinos, $\nu_{R, i}$. The Lagrangian responsible for lepton masses has the form

$$
\mathcal{L}_{\text {Mass }}^{l}=\nu_{R}^{c T} Y_{\nu} \nu_{L}\left\langle H^{0}\right\rangle+l_{R}^{c T} Y_{l} l_{L}\left\langle\bar{H}^{0}\right\rangle-\frac{1}{2} \nu_{R}^{c T} M_{\nu} \nu_{R}^{c}
$$

where $Y_{\nu}, Y_{l}$ are the matrices of Yukawa couplings which give rise to the neutrino and charged lepton Dirac mass matrices respectively and $M_{\nu}$ is the neutrino Majorana mass matrix. We are interested in studying the implications of simultaneous zeros in $Y_{\nu}$ and $Y_{l}$ for observable quantities, i.e. masses, mixing angles and $\mathrm{CP}$ violating phases. For the case of quarks and charged leptons it is easy to do this because the Yukawa couplings are directly related to the mass matrices. For neutrinos, however, the existence of the Majorana masses complicates the connection between the Dirac Yukawa couplings and the neutrino observables. The light neutrino mass matrix, $\mathcal{M}$, is given by the see-saw form

$$
\mathcal{M}=Y_{\nu}^{T} M_{\nu}^{-1} Y_{\nu}
$$

If one tries to reconstruct the complete theory from this effective operator, one finds that in the decoupling process some information has been lost. The complete leptonic Lagrangian depends on twenty one parameters. In an appropriate basis, these can be identified with the three masses in the charged lepton sector, the three masses in the right-handed sector, and fifteen elements in the neutrino Yukawa matrix (nine moduli and six phases). On the other hand, the effective Lagrangian depends on only twelve parameters: three in the charged lepton sector, and six moduli and three phases in the neutrino mass matrix. Therefore, in the decoupling process six moduli and three phases have been lost.

To the end of reconstructing the complete theory it is sometimes convenient to use an alternative form of the see-saw formula, expressing $Y_{\nu}$ in terms of the neutrino mass eigenvalues, mixing angles and $\mathrm{CP}$ violating phases [i, $]$. In the basis in which the Majorana mass matrix and the charged lepton Yukawa matrix are diagonal, the parametrization has the form

$$
Y_{\nu}=D_{\sqrt{M}} R D_{\sqrt{m}} U^{\dagger} /\left\langle H^{0}\right\rangle
$$

where $D_{\sqrt{M}}$ is the diagonal matrix of the square roots of the eigenvalues of $M_{\nu}, D_{\sqrt{m}}$ is the diagonal matrix of the roots of the physical masses, $m_{i}$, of the light neutrinos, $U$ is 
the Maki-Nakagawa-Sakata (MNS) matrix $[\overline{\overline{7}}]$ and $R$ is a $3 \times 3$ orthogonal matrix which parametrizes the residual freedom in $Y_{\nu}$ once the other parameters are fixed. Notice that we have included all the low energy phases in the definition of the matrix $U$, i.e. we have written the MNS matrix in the form $U=V \operatorname{diag}\left(e^{-i \phi / 2}, e^{-i \phi^{\prime} / 2}, 1\right)$, where $\phi$ and $\phi^{\prime}$ are the $\mathrm{CP}$ violating phases and $V$ has the form of the CKM matrix. In this particular parametrization of the see-saw mechanism, the information lost in the decoupling process is encoded in the right handed masses and the $R$ matrix. Incidentally, $M$ depends on three real parameters, that together with the three complex parameters of the matrix $R$ make up the six real and the three complex parameters necessary to match the number of parameters at high and low energies.

These nine unknown parameters prevent us from making predictions from the see-saw mechanism. However, under some well motivated hypotheses on the high energy physics, the number of unknown parameters is reduced, improving the predictability of the seesaw mechanism or even leading to relations among the low energy observables. Some possibilities are:

- Texture zeros in $Y_{\nu}$. The main motivation for this hypothesis comes from the GST relation. However it is also motivated from the theoretical point of view. It may be that an underlying symmetry forbids the appearance of one or more Yukawa couplings, as in the case of family symmetries, where the different matrix elements

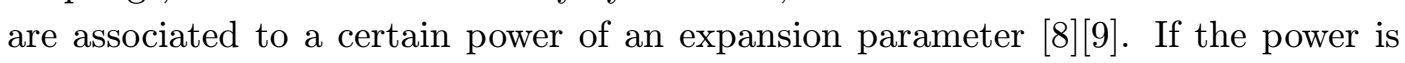
large enough, the suppression of that matrix element will result in a texture zero. Besides, in some supersymmetric scenarios, the holomorphicity of the superpotential could forbid some matrix elements, resulting in a strict texture zero. In any case, it can be checked from eq.( $(2 \overline{2} \cdot 3)$ that one texture zero fixes one of the complex angles in $R$ in terms of the MNS matrix and the light neutrino masses. More texture zeros will further reduce the number of unknown parameters.

- Relations among elements in $Y_{\nu}$. It could occur that an underlying symmetry restricts the fundamental Yukawa couplings to be symmetric, or hermitian, or to have off-diagonal elements antisymmetric. This possibility is also motivated phenomenologically by the success of the GST relation, where symmetric textures were assumed. Under this assumption, the requirement $Y_{\nu i j}=Y_{\nu j i}$ yields three conditions that fix $R$ in terms of the ratio of right-handed masses, the light neutrino masses, and the MNS matrix.

- Decoupling of $\nu_{R, 3}$. The possibility that one of the right-handed neutrinos does not contribute to the neutrino masses and mixing angles underlies many explicit models that reproduce the observed large neutrino mixings [i1 $\left.{ }_{1}^{1} \overline{1}\right]$. In any case, assuming that one of the right-handed neutrinos is much heavier than the other two is an interesting physical possibility that furthermore reduces the number of unknown parameters in the see-saw mechanism. This reduction of unknown parameters should be reflected in the number of parameters of the matrix $R$. To be precise, in the limit in which $M_{3}$ is much heavier than $M_{1}$ and $M_{2}$, the matrix $R$ essentially depends on one single 
complex parameter. The reason is the following. From eq. $\left(\overline{2} \cdot \overline{2}^{3}\right)$ one finds that the elements of the third row of $R$ are given by

$$
R_{3 i}=\frac{(Y U)_{3 i}}{\sqrt{M_{3} m_{i}}}\left\langle H^{0}\right\rangle
$$

Since the numerator is finite and $m_{2}$ and $m_{3}$ are different from zero, $R_{32}$ and $R_{33}$ get smaller and smaller as $M_{3}$ becomes larger and larger. On the other hand, $m_{1} \rightarrow 0$ as $M_{3} \rightarrow \infty$, so the limit for $R_{31}$ is not well defined and might be non-zero. As a matter of fact, the orthogonality of $R$ requires $R_{31}$ to be approximately equal to one. Therefore, in the limit in which $M_{3}$ becomes very heavy, the matrix $R$ takes the form:

$$
R=\left(\begin{array}{ccc}
\propto \sqrt{\frac{1}{M_{3}}} & \cos z & \pm \sin z \\
\propto \sqrt{\frac{1}{M_{3}}} & -\sin z & \pm \cos z \\
\sim 1 & \propto \sqrt{\frac{1}{M_{3}}} & \propto \sqrt{\frac{1}{M_{3}}}
\end{array}\right)
$$

where $z$ is a complex angle and the \pm in the second column has been included to account for the possible reflections in the orthogonal matrix $R$.

In some realistic scenarios, like the ones that we are going to discuss in the following sections, several of these possibilities occur at the same time. This results in a reduction of the number of unknown parameters that will give rise to predictions for the low energy observables. In particular, we will study in detail the leptonic version of the GST relation and the model with two right-handed neutrinos.

\section{The GST relation for the leptonic sector}

We first consider how the analysis proceeds for the case the texture zero is in the $(1,1)$ position of the neutrino Yukawa coupling, in the basis where the charged-lepton Yukawa coupling and the right-handed mass matrix are both diagonal, and when $M_{1,2} / M_{3} \ll$ $m_{2} / m_{3}$. In this case a symmetric structure in the Dirac neutrino mass matrix and a texture zero will lead to a relation between measurable parameters, in particular, for the CHOOZ angle. Later on, we will discuss the effect of the charged lepton sector on the prediction for the $\mathrm{CHOOZ}$ angle.

Following eq.(产.3i]), the condition $\left(Y_{\nu}\right)_{11}=0$ gives $^{2}$

$$
\tan z=-\sqrt{\frac{m_{2}}{m_{3}}} \frac{U_{12}^{*}}{U_{13}^{*}}
$$

From the symmetric constraint $\left(Y_{\nu}\right)_{12}=\left(Y_{\nu}\right)_{21}$ one obtains

$$
\sqrt{\frac{M_{1}}{M_{2}}}=\frac{-\tan z \sqrt{m_{2}} U_{12}^{*}+\sqrt{m_{3}} U_{13}^{*}}{\sqrt{m_{2}} U_{22}^{*}+\tan z \sqrt{m_{3}} U_{23}^{*}} .
$$

\footnotetext{
${ }^{2}$ Here and in what follows we do not include the ambiguity due to the square roots as they can be absorbed in the unknown phases.
} 
Substituting for $\tan z$ leads to the relation

$$
U_{13} \equiv\left|U_{13}\right| e^{i \delta}= \pm i \sqrt{\frac{m_{2}}{m_{3}} U_{12}^{2}+\sqrt{\frac{M_{1}}{M_{2}}} \sqrt{\frac{m_{2}}{m_{3}}} U_{31}^{*} \operatorname{det} U}
$$

This equation is yielding a prediction for the CHOOZ angle in terms of the solar and atmospheric mixing angles, the ratio of neutrino masses, the ratio of the right-handed masses, and the Majorana phase $\phi^{\prime}$. In Figure 1, left plot, we show the prediction for the $\mathrm{CHOOZ}$ angle for different ratios of the right-handed masses. In the plot we have chosen a random distribution for the unmeasured phase $\phi^{\prime}$. One may see that there is a clustering of values within a small range with the $\mathrm{CHOOZ}$ angle near the current bound, $\sin \theta_{13}<0.24$ at $3 \sigma$. This implies that, barring an unnatural cancellation between terms, we expect a large $\mathrm{CHOOZ}$ angle, in the range that would make the long baseline neutrino factory searches for $\mathrm{CP}$ violation feasible. To quantify this we have determined the range of the $\mathrm{CHOOZ}$ angle which includes $95 \%$ of the points, giving $\sin \theta_{13}>0.1$ over the whole range of $M_{1} / M_{2}$.

More interesting than this case is the case where there is a simultaneous texture zero in the $(1,1)$ position in both $Y_{\nu}$ and $Y_{l}$, which truly corresponds to the leptonic version of the GST relation. In [1] ${ }_{1}^{1} \overline{1}_{1}^{i}$ it was shown that the effect from the charged-lepton sector can be included in the analysis by replacing $U \rightarrow W=U_{l} P U$ in eq.( $(\overline{2} \cdot-3 i)$. Here, $W$ is the matrix acting on the left-handed neutrino states needed to diagonalize the neutrino Yukawa matrix, $U_{l}$ is the corresponding one to diagonalize the charged lepton Yukawa matrix, and $P$ is a matrix that appears due to a phase ambiguity because the basis in which the MNS matrix has the standard form can be different from the "symmetry" basis in which the texture zero appears. For the case the charged-lepton Yukawa matrix has off-diagonal elements whose magnitude is approximately symmetric and that, like the quarks, the hierarchy of lepton masses is due to a hierarchical structure in the matrix elements and not due to a cancellation between the different contributions, one has the bounds $\left|U_{l, 23}\right| \leq \sqrt{\frac{m_{\mu}}{m_{\tau}}}$, $\left|U_{l, 12}\right| \leq \sqrt{\frac{m_{e}}{m_{\mu}}},\left|U_{l, 13}\right| \leq \sqrt{\frac{m_{e}}{m_{\tau}}}$. In practice, the magnitudes of $\left|U_{l, 23}\right|$ and $\left|U_{l, 13}\right|$ are so small that they do not affect the mixing coming from the neutrino sector. However, $\left|U_{l, 12}\right|$ close to the upper bound does give a significant contribution to the $\mathrm{CHOOZ}$ angle. In fact, this bound is saturated for a symmetric charged-lepton Yukawa matrix with a $(1,1)$ texture zero. Therefore, neglecting the contributions from the right-handed sector, the leptonic version of the GST relation reads:

$$
U_{13} \equiv\left|U_{13}\right| e^{i \delta}=-e^{i\left(\alpha_{2}-\alpha_{1}\right)} \sqrt{\frac{m_{e}}{m_{\mu}}} U_{23} \pm i \sqrt{\frac{m_{2}}{m_{3}} U_{12}^{2}+\sqrt{\frac{M_{1}}{M_{2}}} \sqrt{\frac{m_{2}}{m_{3}}} U_{31} e^{-i\left(\beta+2 \alpha_{1}\right)}} .
$$

In this formula, $\alpha_{1}, \alpha_{2}$ and $\beta$ are unknown phases. As in the previous case, we choose a random distribution of these phases, and plot the distribution of the resulting points. This is shown in Figure 1, right plot.

In Figure 2 we plot the distribution for the $\mathrm{CP}$ violating phase combination $\sin (\delta-$ $\left.\phi^{\prime} / 2\right)$. This is the CP violating phase relevant to neutrinoless double beta decay. We see that $\sin \left(\delta-\phi^{\prime} / 2\right)$ clusters near its maximal value. In this case the $95 \%$ cutoff implies $\sin \left(\delta-\phi^{\prime} / 2\right)>0.4$. 

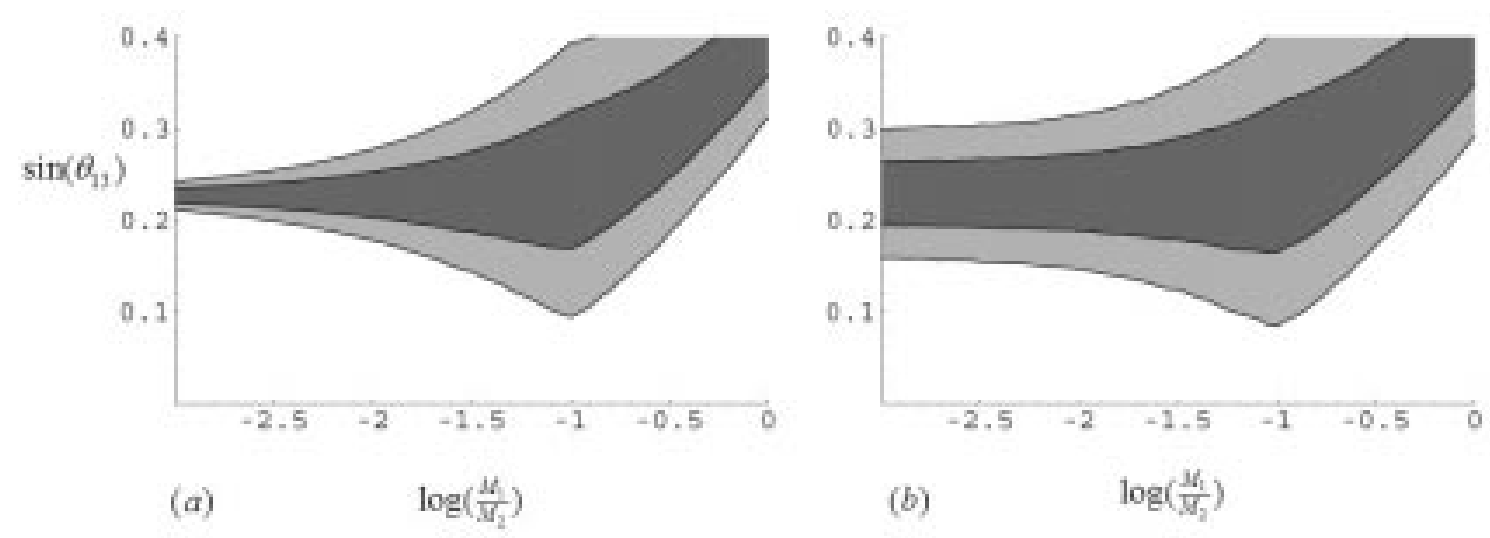

Figure 1: Prediction for the $\mathrm{CHOOZ}$ angle from a symmetric neutrino Yukawa matrix with a zero in the $(1,1)$ position, assuming that there is no contribution from the charged lepton sector (left), and that there is a simultaneous $(1,1)$ texture zero in the charged lepton Yukawa coupling (right).
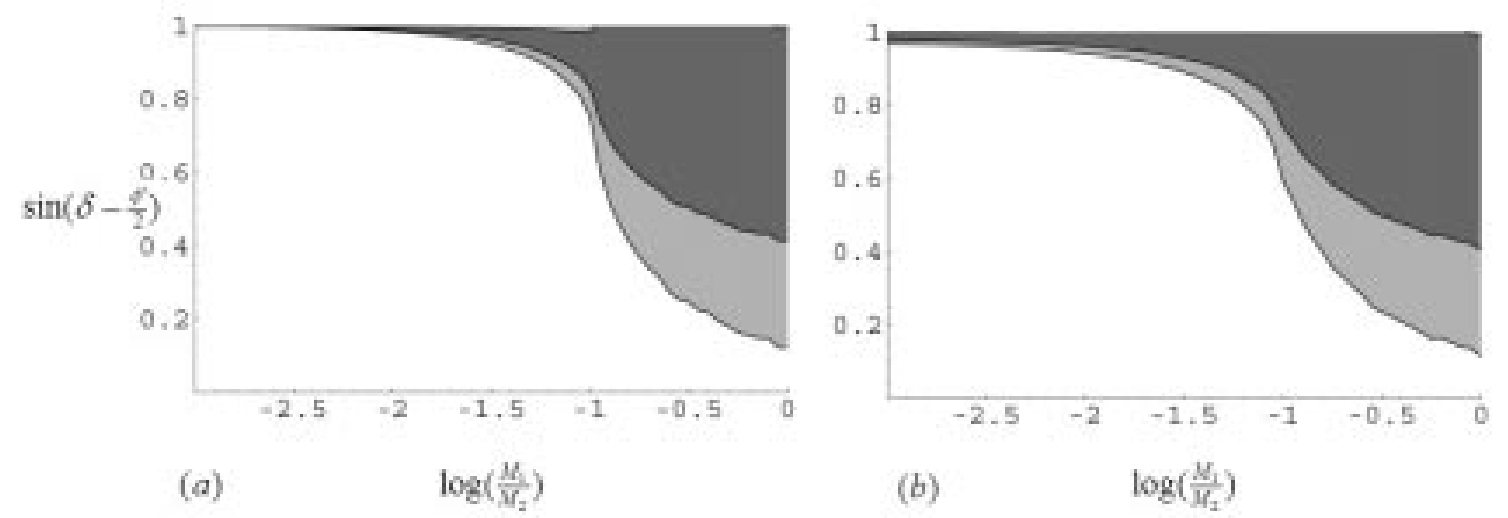

Figure 2: The $\nu 0 \beta \beta \mathrm{CP}$ violating phase, for the same cases as in Figure 1.

\section{The two right-handed neutrino model}

This model is able to accommodate all the neutrino observations, but depends at high energies on less parameters than the conventional see-saw model. This model has attracted

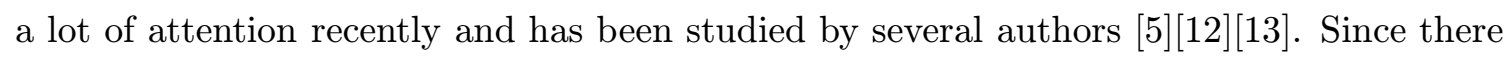
are only two mass scales, one of the light neutrinos is predicted to be exactly massless. This implies in particular that there is only one Majorana phase difference between the light neutrinos. Notice that the remaining two mass scales are enough to reproduce the measured solar and atmospheric neutrino mass splittings. In an appropriate basis, the two right-handed neutrino (2RHN) model is defined by three charged lepton masses, two right-handed masses and six moduli and three phases in the neutrino Yukawa coupling. Therefore, the model with two right-handed neutrinos depends at high energies on four moduli and three phases less than the see-saw model with three right handed neutrinos 
(3RHN). This reduction of parameters should be translated into a simplification of the matrix $R$. It is clear that the $2 \mathrm{RHN}$ model corresponds to the strict decoupling, $M_{3} \rightarrow \infty$, of the 3RHN model. Then, from eqs. $(\overline{2} . \overline{3})$ and $(\overline{2} . \overline{2} . \overline{1})$ one obtains that the different elements of the neutrino Yukawa coupling are:

$$
\begin{aligned}
& \mathbf{Y}_{\nu 1 i}=\sqrt{M_{1}}\left(\sqrt{m_{2}} \cos z U_{i 2}^{*} \pm \sqrt{m_{3}} \sin z U_{i 3}^{*}\right) /\left\langle H^{0}\right\rangle \\
& \mathbf{Y}_{\nu 2 i}=\sqrt{M_{2}}\left(-\sqrt{m_{2}} \sin z U_{i 2}^{*} \pm \sqrt{m_{3}} \cos z U_{i 3}^{*}\right) /\left\langle H^{0}\right\rangle
\end{aligned}
$$

where $i=1,2,3$. The unknown complex parameter $z$ encodes the real parameter and the phase necessary to match the total number of parameters at high energies and at low energies in the $2 \mathrm{RHN}$ model.

A word of caution is in order concerning the relation between the decoupling limit of the 3 RHN case and the 2 RHN case. In the 3 RHN case the Yukawa couplings $\mathbf{Y}_{\nu 3 i}$ are not necessarily negligible due to the factor $\sqrt{M_{3}}$ appearing in eq. $\left(\overline{2}_{2}^{2} \cdot \overline{3}_{1}\right)$, and could produce some effect at low energies through the radiative corrections. Whether or not they are depends on the magnitude of the elements $R_{3, j}, j=2,3$ which vanish like $1 / \sqrt{M_{3}}$. The decoupling limit corresponds to the case that the third Majorana mass is at, or above, the cutoff scale so that the third neutrino does not contribute, even via radiative corrections.

With this tools, it is straightforward to compute predictions at low energies from texture zeros in the two right handed neutrino model. In what follows, we will assume that the texture zeros appear in the basis where the charged lepton Yukawa couplings and the right-handed mass matrix are diagonal. This is not the most general possibility, nor the most attractive from the physical point of view. However, for simplicity we will show only the results for this particular case, and we refer the reader to reference [i] $\overline{1} \overline{3}]$ ] for the details of the most general case.

\subsection{Predictions from models with one texture zero}

One texture zero in the neutrino Yukawa coupling fixes the unknown parameter $z$. From

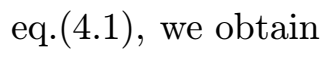

$$
\tan z=\mp \sqrt{\frac{m_{2}}{m_{3}}} \frac{U_{i 2}^{*}}{W_{i 3}^{*}}
$$

when $Y_{1 i}^{T Z}=0$, and

$$
\tan z= \pm \sqrt{\frac{m_{3}}{m_{2}}} \frac{U_{i 3}^{*}}{W_{i 2}^{*}}
$$

when $Y_{2 i}^{T Z}=0$. With only these hypotheses, there are no predictions for the low energy parameters. However, it has implications for thermal leptogenesis [i $[17]$, assuming that the lightest Majorana state dominates [i $\left[\begin{array}{l}1 \\ 5\end{array}\right]$. In this case the asymmetry is given by

$$
\begin{aligned}
\epsilon & \simeq-\frac{3}{8 \pi} \frac{M_{1}}{\left\langle H^{0}\right\rangle^{2}} \frac{\operatorname{Im}\left(\cos ^{2} z m_{2}^{2}+\sin ^{2} z m_{3}^{2}\right)}{m_{2}\left|\cos ^{2} z\right|+m_{3}\left|\sin ^{2} z\right|} \\
& =-\frac{3}{8 \pi} \frac{M_{1}}{\left\langle H^{0}\right\rangle^{2}} \frac{\left(m_{3}^{2}-m_{2}^{2}\right) \operatorname{Im}\left(\sin ^{2} z\right)}{m_{2}\left|\cos ^{2} z\right|+m_{3}\left|\sin ^{2} z\right|}
\end{aligned}
$$


Since $\left|\epsilon_{\max }\right|=\frac{3}{8 \pi} \frac{M_{1} m_{3}}{\left\langle H^{0}\right\rangle^{2}}[\underline{1} \overline{1} \overline{\underline{6}}]$ ] $]$, we have

$$
\frac{\epsilon}{\left|\epsilon_{\max }\right|} \simeq-\frac{\operatorname{Im}\left(\sin ^{2} z\right)}{\left|\sin ^{2} z\right|+\frac{m_{2}}{m_{3}}\left|\cos ^{2} z\right|}
$$

Note that $\epsilon$ depends only on $\tan z$, which is in turn fixed by the texture zero. Whether this asymmetry can lead to the observed baryon asymmetry depends on the subsequent washout. This is characterized by the parameter $\widetilde{m}_{1}\left[{ }_{1}^{1} \overline{\overline{1}} \overline{1}\right]$. It is given by

$$
\widetilde{m}_{1}=m_{2}\left|\cos ^{2} z\right|+m_{3}\left|\sin ^{2} z\right|
$$

Notice that $\widetilde{m}_{1} \geq m_{2}$, so washout effects are expected to be very efficient, potentially in conflict with the upper bound following from the condition that washout is acceptable. Furthermore, the parameters that maximize the CP asymmetry could not necessarily correspond to those that minimize $\widetilde{m}_{1}$. It can be checked that if the washout effects are minimal, the CP asymmetry vanishes. Physically, this scenario corresponds to the case in which the heaviest light neutrino state is dominated by the heaviest right-handed neutrino state $(\cos z \simeq 1)$. On the other hand, when the CP asymmetry is close to maximal, $\widetilde{m}_{1}$ must be very large and successful leptogenesis would require a large right-handed neutrino mass, $M_{1} \geq 10^{11} \mathrm{GeV}$. In particular, this is the case for the limit in which the heaviest light neutrino state is dominated by the heaviest right-handed neutrino state $(\cos z \simeq 0)$. In a supersymmetric model with gravity mediated supersymmetry breaking, the reheat temperature pointed by these large values of the right-handed mass could be in conflict with the gravitino overproduction constraints. However, this problem can be circumvented in other supersymmetry breaking mediation scenarios, such as gauge mediation, where the gravitino can be much lighter.

An interesting issue that has been discussed extensively in the literature concerns the connection of leptogenesis and low energy observables [i] $\left.{ }_{1}^{1}\right]$. This connection is clear only when $U_{e} \simeq 1$ and $V_{\nu} \simeq 1$, otherwise some unmeasurable parameters in the charged-lepton sector or the right-handed sector enter into play. In particular, it has been discussed the correlation between the sign of the baryon asymmetry and the $\mathrm{CP}$ violation at low energies. We find that one texture zero is enough to establish such connection, since the sign of the $\mathrm{CP}$ asymmetry is determined by minus the argument of $\tan ^{2} z$, which is in turn fixed just with one texture zero. For instance, when the texture zero appear in the $(1,2)$ or $(1,3)$ position,

$$
\begin{aligned}
\frac{\epsilon_{1}}{\left|\epsilon_{\max }\right|} & \simeq-\frac{\sin \phi^{\prime}}{1+\left|\frac{U_{i 3}}{U_{i 2}}\right|^{2}} \simeq-\frac{\cos ^{2} \theta_{12}}{1+\cos ^{2} \theta_{12}} \sin \phi^{\prime} \\
\tilde{m}_{1} & \simeq m_{2}\left(1+\left|\frac{U_{i 2}}{U_{i 3}}\right|^{2}\right) \simeq m_{2}\left(1+\cos ^{2} \theta_{12}\right),
\end{aligned}
$$

where $i=2,3$. On the other hand, when it appears in the $(2,2)$ or $(2,3)$ position,

$$
\begin{aligned}
\frac{\epsilon_{1}}{\left|\epsilon_{\max }\right|} & \simeq \frac{m_{2}}{m_{3}}\left|\frac{U_{i 2}}{U_{i 3}}\right|^{2} \sin \phi^{\prime} \simeq \frac{m_{2}}{m_{3}} \cos ^{2} \theta_{12} \sin \phi^{\prime} \\
\widetilde{m}_{1} & \simeq m_{3}
\end{aligned}
$$


where we have used a maximal value for the atmospheric angle. Finally, the case when the texture zero appears in the first column deserves some more careful analysis, since it involves the CHOOZ angle which has not been measured. The expressions are rather

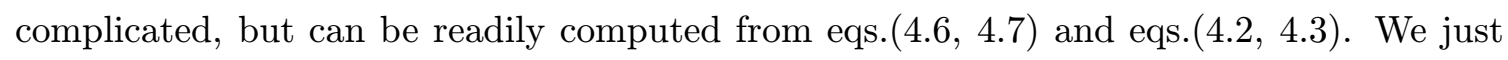
show the numerical results in Figure 3, where we plot the CP asymmetry divided by $\sin \left(2 \delta-\phi^{\prime}\right)$, to show better the connection between the sign of the CP asymmetry and the low energy CP violation. The lepton asymmetry and $\sin \left(2 \delta-\phi^{\prime}\right)$ have the same sign for the $(1,1)$ texture zero and opposite for the $(2,1)$ texture zero (the sign of the baryon asymmetry is opposite to the sign of the lepton asymmetry).

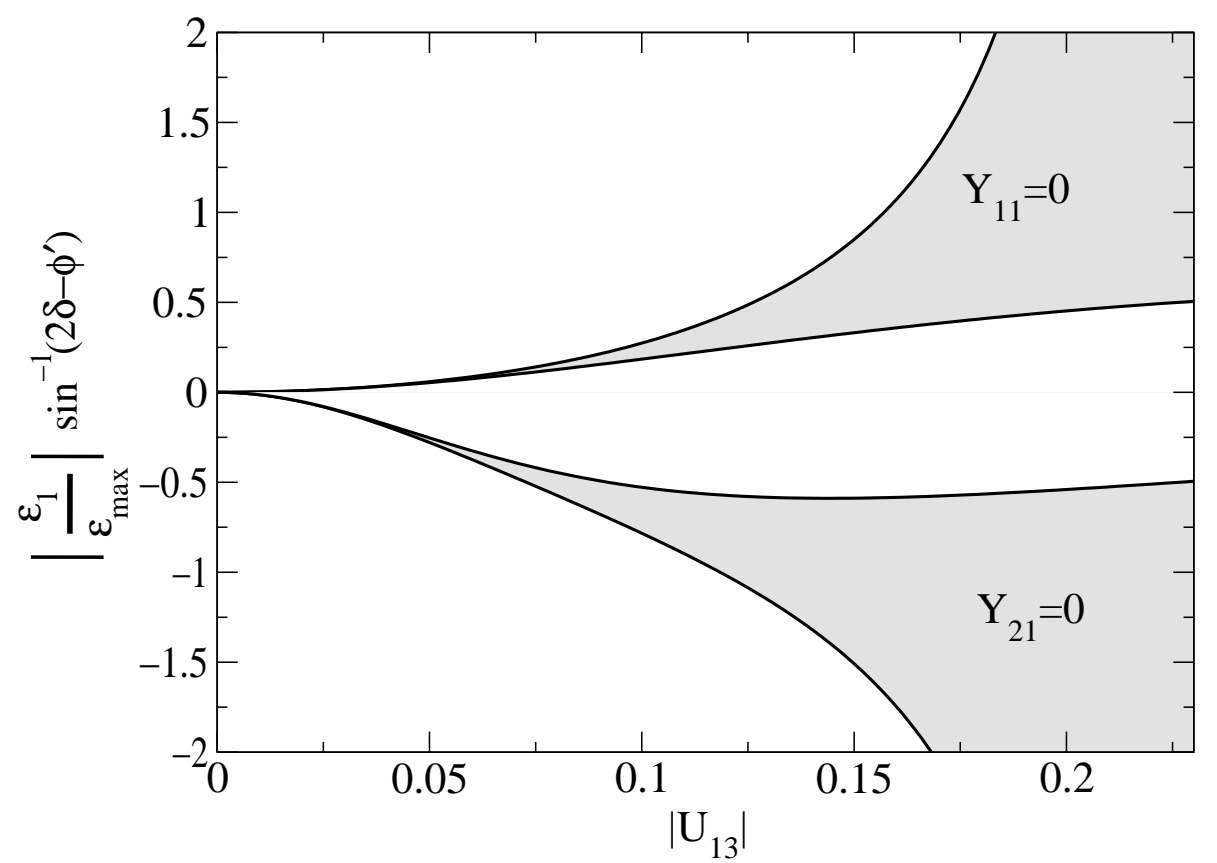

Figure 3: Connection between the sign of the $\mathrm{CP}$ asymmetry and the sign of the low energy CP violation, for the scenario with two right-handed neutrinos and a $(1,1)$ or $(2,1)$ texture zero.

\subsection{Predictions from models with two texture zeros}

We can write now the predictions from two texture zeros in the neutrino Yukawa matrix. When the two texture zeros appear in the same row, $Y_{1 i}^{T Z}=0, Y_{1 j}^{T Z}=0$ or $Y_{2 i}^{T Z}=0$, $Y_{2 j}^{T Z}=0$, then

$$
\epsilon_{i j k} U_{k 1}=0 \text {. }
$$

On the other hand, when the texture zeros appear in different rows, $Y_{1 i}^{T Z}=0, Y_{2 j}^{T Z}=0$, the following relation holds:

$$
U_{i 3} U_{j 3}+\frac{m_{2}}{m_{3}} U_{i 2} U_{j 2}=0
$$

In Table $\underline{I}_{1}^{\overline{1}}$ we show the predictions at low energies for all the fifteen possible Yukawa matrices with two texture zeros. Only five of them are allowed by present experiments, 
namely textures IV, VII, and VIII. The matrix with texture zeros in the same column leads to the prediction for the $\mathrm{CHOOZ}$ angle $s_{13} \simeq \sqrt{\frac{m_{2}}{m_{3}}} \sin \theta_{\text {sol }} \simeq 0.22$, which is marginally allowed, and a $\mathrm{CP}$ violating phase $\delta \simeq \phi^{\prime} / 2$. On the other hand, the other four possibilities yield $s_{13} \simeq \frac{m_{2}}{2 m_{3}} \sin 2 \theta_{\text {sol }} \simeq 0.08$ and a phase $\delta \simeq \phi^{\prime}$, for the textures with zeros in the first and third columns, or $\delta \simeq \phi^{\prime}+\pi$, for the textures with zeros in the first and second columns.

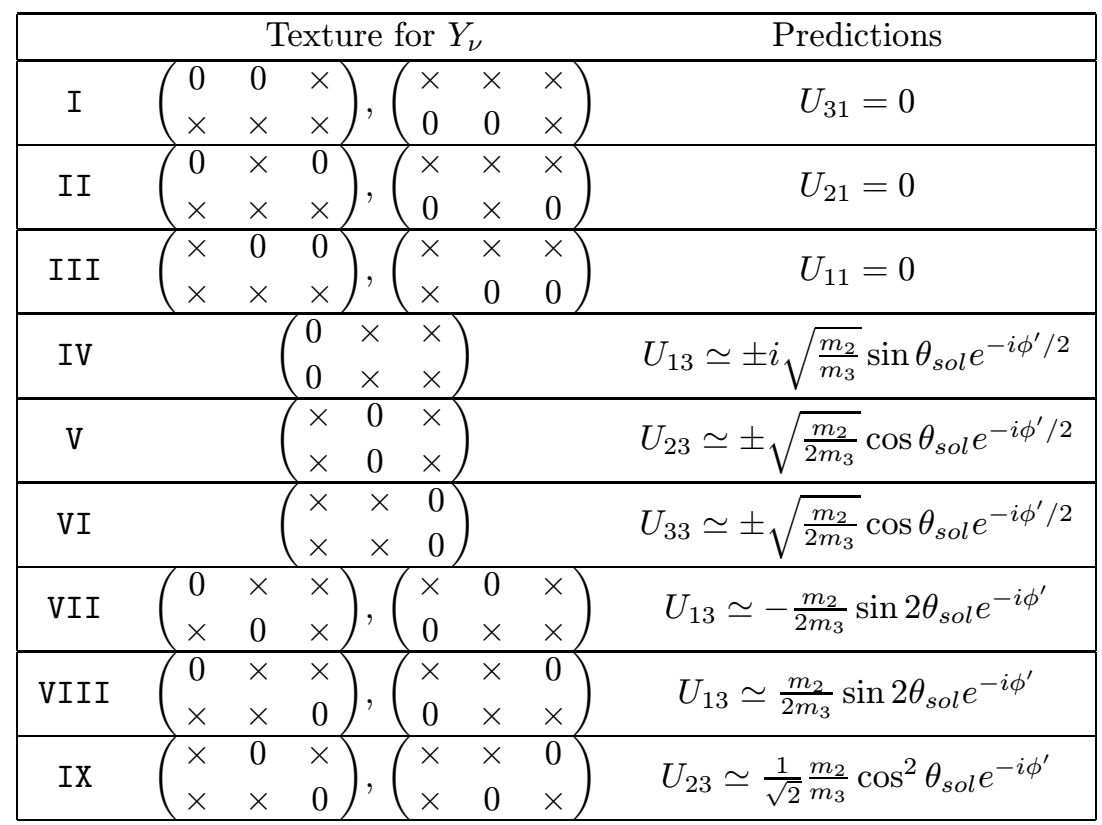

Table 1: Predictions following from the various two texture zero structures.

\section{Conclusions}

The analysis of the neutrino phenomenology coming from the see-saw mechanism is always limited by the lack of predictability of this otherwise interesting scenario. However, under some well motivated hypotheses on the high energy physics, the see-saw mechanism can become predictive. In this work, we have explored two scenarios where it is indeed possible to make predictions from the see-saw mechanism, namely, the scenario with symmetric textures and a simultaneous $(1,1)$ zero in the Yukawa matrices, and the scenario with only two right-handed neutrinos and one or two texture zeros.

In the first case, we have derived a leptonic version of the Gatto-Sartori-Tonin relation. We have obtained a lower bound on the CHOOZ angle $s_{13} \geq 0.1$ at $95 \%$ c.l., that should make feasible the search for $\mathrm{CP}$ violation at the long baseline neutrino factory. We have also shown that the phase combination relevant for neutrinoless double beta decay is close to the maximal value.

Concerning the model with two right-handed neutrinos, we have found a connection between leptogenesis and low energy $\mathrm{CP}$ violation with just one texture zero, and we have shown the predictions for all the fifteen Yukawa matrices with two texture zeros, in the case 
when these zeros appear in the basis where the charged lepton Yukawa coupling and the right-handed mass matrix are diagonal. Among those, there are only five neutrino Yukawa matrices that yield predictions allowed by experiments, namely $s_{13} \simeq 0.22$ or $s_{13} \simeq 0.08$, depending on the positions of the texture zeros.

\section{References}

[1] R. Gatto, G. Sartori and M. Tonin, Weak Selfmasses, Cabibbo Angle, And Broken SU(2) X SU(2), Phys. Lett. B 28 (1968) 128.

R. J. Oakes, SU(2) X SU(2) Breaking And The Cabibbo Angle,

[2] P. Ramond, R. G. Roberts and G. G. Ross, Stitching the Yukawa quilt, Nucl. Phys. B 406 (1993) 19

[3] R. G. Roberts, A. Romanino, G. G. Ross and L. Velasco-Sevilla, Precision test of a fermion mass texture, Nucl. Phys. B 615 (2001) 358

[4] M. Gell-Mann, P. Ramond and R. Slansky, Proceedings of the Supergravity Stony Brook Workshop, New York 1979, eds. P. Van Nieuwenhuizen and D. Freedman;

T. Yanagida, Proceedinds of the Workshop on Unified Theories and Baryon Number in the Universe, Tsukuba, Japan 1979, ed.s A. Sawada and A. Sugamoto;

R. N. Mohapatra, G. Senjanovic, Phys.Rev.Lett. 44 (1980)912, ibid. Phys.Rev. D23 (1981) 165 ;

S. L. Glashow, The Future Of Elementary Particle Physics, In ${ }^{*}$ Cargese 1979, Proceedings, Quarks and Leptons*, 687-713 and Harvard Univ.Cambridge - HUTP-79-A059

(79,REC.DEC.) $40 p$.

[5] P. H. Frampton, S. L. Glashow and T. Yanagida, Cosmological sign of neutrino CP violation, Phys. Lett. B 548 (2002) 119

[6] J. A. Casas and A. Ibarra, Oscillating neutrinos and $m u \rightarrow e$, gamma, Nucl. Phys. B 618 (2001) 171

[7] Z. Maki, M. Nakagawa and S. Sakata, Remarks On The Unified Model Of Elementary Particles, Prog. Theor. Phys. 28 (1962) 870.

[8] C. D. Froggatt and H. B. Nielsen, Hierarchy Of Quark Masses, Cabibbo Angles And CP Violation, Nucl. Phys. B 147 (1979) 277.

[9] L. E. Ibanez and G. G. Ross, Fermion Masses And Mixing Angles From Gauge Symmetries, Phys. Lett. B 332 (1994) 100

[10] S. F. King, Atmospheric and solar neutrinos with a heavy singlet, Phys. Lett. B 439 (1998) 350

G. Altarelli, F. Feruglio and I. Masina, Large neutrino mixing from small quark and lepton mixings, Phys. Lett. B 472 (2000) 382

A. Y. Smirnov, Seesaw Enhancement Of Lepton Mixing, Phys. Rev. D 48 (1993) 3264

[11] A. Ibarra and G. G. Ross, Neutrino properties from Yukawa structure, Phys. Lett. B 575 (2003) 279.

[12] M. Raidal and A. Strumia, Predictions of the most minimal see-saw model, Phys. Lett. B $\mathbf{5 5 3}(2003) 72$ 
V. Barger, D. A. Dicus, H. J. He and T. j. Li, Structure of cosmological CP violation via neutrino seesaw, arXiv:hep-ph/0310278.

W. l. Guo and Z. z. Xing, Calculable CP-violating phases in the minimal seesaw model of leptogenesis and neutrino mixing arXiv:hep-ph/0310326.

[13] A. Ibarra and G. G. Ross, Neutrino Phenomenology - the case of the two right-handed neutrinos. arXiv:hep-ph/0312138.

[14] M. Fukugita and T. Yanagida, Phys. Lett. B 174 (1986) 45.

[15] W. Buchmuller, P. Di Bari and M. Plumacher, The neutrino mass window for baryogenesis, Nucl. Phys. B 665 (2003) 445

[16] S. Davidson and A. Ibarra, A lower bound on the right-handed neutrino mass from leptogenesis, Phys. Lett. B 535 (2002) 25

[17] M. Plumacher, Baryogenesis and lepton number violation, Z. Phys. C 74 (1997) 549.

[18] S. Davidson and A. Ibarra, Leptogenesis and low-energy phases Nucl. Phys. B 648 (2003) 345 S. Davidson, From weak-scale observables to leptogenesis, JHEP 0303 (2003) 037

S. Pascoli, S. T. Petcov and W. Rodejohann, On the connection of leptogenesis with low energy CP violation and LFV charged lepton decays Phys. Rev. D 68 (2003) 093007 G. C. Branco, R. Gonzalez Felipe, F. R. Joaquim, I. Masina, M. N. Rebelo and C. A. Savoy, Minimal scenarios for leptogenesis and CP violation Phys. Rev. D 67 (2003) 073025 